\title{
A New Sound Absorption Improvement Strategy under Low Frequency for a Rigid Micro-perforated Panel Absorbers
}

\author{
Shumei Liu \\ Department of Automation \\ University of Science and Technology of China \\ Hefei, China \\ liu0558@mail.ustc.edu.cn \\ Ping Li \\ Department of Automation \\ University of Science and Technology of China \\ Hefei, China \\ liping89@mail.ustc.edu.cn
}

\author{
Zibo Li \\ Department of Automation \\ University of Science and Technology of China \\ Hefei, China \\ 11z1b0@126.com
}

\author{
Likai Zhu \\ Institute of Intelligent Machines \\ Chinese Academy of Sciences \\ Hefei, China \\ lkzhu@iim.ac.cn
}

\begin{abstract}
A micro-perforated panel absorbers (MPPs) is one of the most promising alternatives among the various types of the next-generation sound absorbers. However, the low frequency noise is difficult to tackle using the general MPPs. According to the high absorption performance of the combination of the membrane absorbers with MPPs and a finite flexible micro-perforated panel backed by an air cavity, the experimental study on the combination of a rigid and a finite flexible micro-perforated panel is carried out to reduce the noise in the low frequency. The results indicate that the resonance shifts to the low frequency. Meanwhile, the peak value becomes higher in the absorption curve of a conventional MPPs, which surface is attached to a finite flexible MPPs or a membrane, in comparison with the normal MPPs. These data demonstrate the great potential of the combination a rigid MPPs with a flexible MPPs in low frequency noise reduction.
\end{abstract}

Keywords-micro-perforated panel; low frequency; finite flexible; membrane; sound absorption capability

\section{INTRODUCTION}

The micro-perforated panel absorbers (MPPs) with sub-millimeter holes were first proposed by Maa in 1975[1] and since then they have been studied theoretically and experimentally. The MPPs are recognized as the most attractive alternatives of the next-generation sound absorbing materials. The basic construction of a MPP absorber consists of a thin flat panel perforated with a large number of sub-millimeter perforations fitted in front of a rigid backing wall with an air space between them.

A typical MPPs can offer a peak sound absorption around the resonance frequency, however, its sound absorption capability is usually not quite enough for a practical applications especially in low frequency noise space, such as the noise in the air conditioning system and engine facility[2]. In order to achieve optimal enhancement in the sound absorption properties of MPPs in low frequency, there has been several noticeable developments. Fuchs $[3,4]$ attempted to improve the sound absorption performance through covering a thin membrane in the front side of MPPs, and their sound absorption properties were experimentally studied. Then the theory of the combination of a membrane absorbers and MPPs was given by Shengwo Sheng [5] and J. Kang [6]. Lin et al. [7] has proposed a method of adding porous sound-absorbing materials in the cavity to obtain a wide band sound absorber. Y. J. Qian [8] found that the absorption peaks of the composite structures are shifted to low frequency compared with the experimental results of MPPs before surface modification using super-aligned carbon nanotube (SACNT) arrays, and the absorption peaks are gradually shifted to low frequency with the increase of the length of SACNT arrays. Furthermore, an additional sound absorption peak was unexpectedly found around the lowfrequency range in the absorption test of a microperforated panel conducted by Lee and Swenson [9]. For expanding the absorption bandwidth of the MPPs, especially in the low frequency, Y. Y. Lee et al. [10] has presented a theoretical model for predicting the absorption coefficient of an absorber, considering the two effects of micro-perforation and finite flexible panel vibration, which show good agreement with the measurements. Thus a flexible MPPs combined with a rigid MPPs may have great potential to improve the absorption ability of typical MPPs, which made the flexible MPPs installation easier and more stable.

The purpose of this study is to examine the effects of the combination of a flexible and a rigid MPPs on acoustic properties of typical MPPs. It is generally known that compared to ordinary MPPs, a flexible MPPs is a kind of well absorption performance in low frequency. In this work, a thin membrane or micro-perforated membrane or flexible MPPs was covered in the front side of MPPs, 
experimental results of the normal sound absorption coefficient of MPPs covered with a thin membrane and flexible MPPs or not are measured according to standard: ISO 10534-1:1996 “Acoustics -- Determination of sound absorption coefficients and impedance in impedance tubes -- Part 1: Method using standing wave ratio" [11].

\section{THEORY}

For an open weave textile or a micro-perforated membrane or a flexible MPPs backed by an air space, the acoustic impedance of such a structure can be obtained using the impedance type of electro-acoustic analogy[6]. Fig. 1 shows the equivalent circuit of a single layer of flexible MPPs mounted at distance D $(\mathrm{mm})$ from a rigid wall, where $R_{h}$ and $M_{h}$ are the specific acoustic resistance and reactance of the apertures of flexible MPPs, $R_{b}$ and $M_{b}$ are the specific acoustic resistance and reactance of the flexible panel. $\rho \mathrm{c}$ is the internal resistance of air [1] and $z_{D}$ is the impedance of the air space between MPPs and the rigid wall, $z_{D}$ can be expressed as:

$$
z_{D}=-\mathrm{j} \rho \mathrm{c} \cdot \cot (\omega D / c) \text {. }
$$

where $\omega=2 \pi f, f$ is the angular frequency of incident acoustic wave. From the equivalent circuit of this structure, we can obtain the acoustic impedance of the whole system. The normal specific acoustic impedance of the flexible panel normalized by $\rho \mathrm{c}$ can be calculated:

$$
z_{b}=\frac{R_{b}+j M_{b}}{\rho c}=r_{b}+j \omega m_{b} .
$$

where $r_{b}$ is the normalized specific acoustic resistance of the flexible panel, $m_{b}$ is the normalized specific acoustic reactance of the flexible panel, $m_{b}=m_{b 0} / \rho c, m_{b 0}$ is the surface density of the flexible panel $\left(\mathrm{kg} / \mathrm{m}^{2}\right)$. According to Maa's model, for normal incidence, the normalized specific acoustic impedance $z_{h}$ of the apertures of the flexible panel can be expressed as:

$$
z_{h}=\frac{R_{h}+j M_{h}}{\rho c}=r_{h}+j \omega m_{h} .
$$

$r_{h}$ and $m_{h}$ are respectively equivalent to the normalized specific acoustic resistance and normalized acoustic mass of typical MPPs.

with

$$
\begin{aligned}
& r_{h}=\frac{32 \mu t}{\sigma c d^{2}}\left(\sqrt{1+\frac{k^{2}}{32}}+\frac{\sqrt{2} k d}{8 t}\right) . \\
& m_{h}=\frac{t}{\sigma c}\left(1+\frac{1}{\sqrt{9+k^{2} / 2}}+\frac{0.85 d}{t}\right) .
\end{aligned}
$$

with

$$
k=\sqrt{\frac{\omega}{\mu}} \frac{d}{2} .
$$

where $\mu$ is kinematic viscosity constant of air, $t$ is the

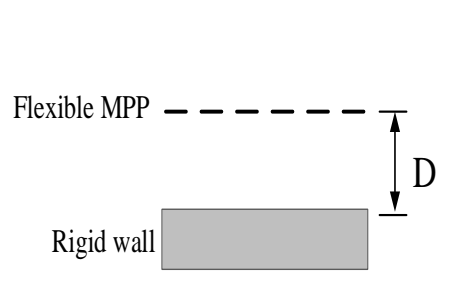

(a)

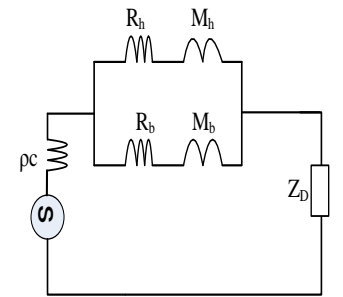

(b)
Figure 1. A structure of flexible MPPs and the equivalent circuit of this structure.

flexible panel thickness ( $\mathrm{mm}), d$ is the aperture diameter $(\mathrm{mm}), \sigma$ is the aperture ratio, and $b$ is the distance between adjacent aperture centres $(\mathrm{mm})$.

According to the equivalent circuit in Fig. 1(b), the normalized specific acoustic impedance of the whole structure can be calculated by

$$
z=\frac{z_{h} z_{b}}{z_{h}+z_{b}}+z_{D} .
$$

For normal incidence, the absorption coefficient is derived by

$$
\alpha=\frac{4 \operatorname{Re}(z)}{[1+\operatorname{Re}(z)]^{2}+[\operatorname{Im}(z)]^{2}} .
$$

A combination of a flexible MPP absorber and a typical MPP absorber can be expected that the sound absorption will be resulted from the combination of those from individual materials and sizes of holes which will make it has great potential to achieve a broader absorption frequency range. Fig. 2 shows the structure of combination of a flexible MPPs and a typical MPPs and the equivalent circuit of this structure. Where $R_{h 1}, M_{h 1}, R_{h 2}$ and $M_{h 2}$ are the specific acoustic resistance and reactance of the apertures of flexible MPPs and rigid MPPs respectively, $R_{b}$ and $M_{b}$ are the specific acoustic resistance and reactance of the flexible panel.

The same to (3):

$$
\begin{gathered}
z_{h 1}=\frac{R_{h 1}+j M_{h 1}}{\rho c}=r_{h 1}+j \omega m_{h 1} . \\
z_{h 2}=\frac{R_{h 2}+j M_{h 2}}{\rho c}=r_{h 2}+j \omega m_{h 2} .
\end{gathered}
$$

with

$$
\begin{gathered}
r_{h 1}=\frac{32 \mu t_{1}}{\sigma_{1} c d_{1}^{2}}\left(\sqrt{1+\frac{k_{1}^{2}}{32}}+\frac{\sqrt{2} k_{1} d_{1}}{8 t_{1}}\right) . \\
m_{h 1}=\frac{t_{1}}{\sigma_{1} c}\left(1+\frac{1}{\sqrt{9+k_{1}^{2} / 2}}+\frac{0.85 d_{1}}{t_{1}}\right) .
\end{gathered}
$$

with

$$
\begin{aligned}
& k_{1}=\sqrt{\frac{\omega}{\mu}} \frac{d_{1}}{2} . \\
& k_{2}=\sqrt{\frac{\omega}{\mu}} \frac{d_{2}}{2} .
\end{aligned}
$$




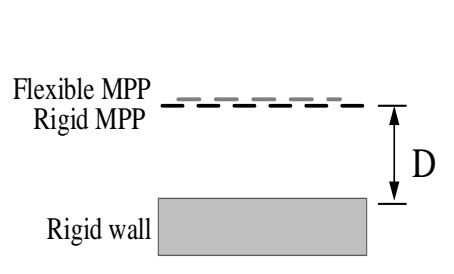

(a)

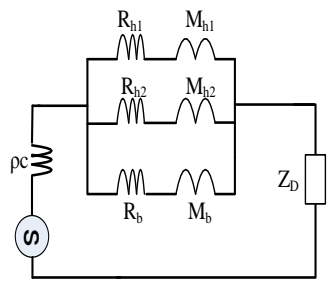

(b)
Figure 2. A structure of combination of a flexible MPPs and a typical MPPs and the equivalent circuit of this structure.

For normal incidence, where $z_{h 1}$ and $z_{h 2}$ are respectively the normalized specific acoustic impedance of the apertures of flexible MPPs and rigid MPPs. $r_{h 1}, m_{h 1}$, $r_{h 2}$ and $m_{h 2}$ are respectively the normalized specific acoustic resistance and normalized acoustic mass of flexible MPPs and rigid MPPs. Where $\mu$ is kinematic viscosity constant of air, $t_{1}, d_{1}, \sigma_{1}$ and $b_{1}$ are the structure parameters of flexible MPPs, $t_{2}, d_{2}, \sigma_{2}$ and $b_{2}$ are the structure parameters of rigid MPPs, and the meaning of them is the same as to (4) and (5).

According to the equivalent circuit in Fig. 2(b), the normalized specific acoustic impedance of the whole structure can be calculated by

$$
z_{\text {total }}=\frac{z_{h 1} z_{h 2} z_{b}}{z_{h 2} z_{b}+z_{h 1} z_{b}+z_{h 1} z_{h 2}}+z_{D} .
$$

For normal incidence, the absorption coefficient is derived by

$$
\alpha=\frac{4 \operatorname{Re}\left(z_{\text {total }}\right)}{\left[1+\operatorname{Re}\left(z_{\text {total }}\right)\right]^{2}+\left[\operatorname{Im}\left(z_{\text {total }}\right)\right]^{2}}
$$

\section{FABRICATION OF MPPS WITH FLEXIBLE OR RIGID MATERIAL}

For the experimental investigation, MEMS technology or needle-punching and PDMS casting method were applied to fabricate the flexible MPPs, a rigid MPPs was fulfilled by means of machining. The MPPs made from PDMS is widely applied in micro-fluidic chips to fabricate structures on microscale [12]. PDMS is highly transparent and is characterized by viscoelastic, which could flow into any structure and be shaped in mold easily. The design parameters are listed in Table I and pictures of some of the specimens are shown in Fig. 3. In Table $\mathrm{I}, t, d, b$ and $D$ have the same meaning as those in Fig. 1(a), $\sigma$ is the perforation ratio. Fig. 3(a) showed the photo of 2\# specimens which was fabricated by Haoyue Ping who is in Institute of Electronics, Chinese Academy of Sciences, Beijing, China using the way of MEMS technology and PDMS casting. Fig. 3(b) gives the picture of 3\# specimens which was fabricated by needle-punching and PDMS casting method.

According to Maa's model [1], the normalized acoustic mass of MPPs depends mainly on its perforation ratio only as illustrated in (4) and (5), while the normalized specific acoustic resistance of MPPs varies also inversely with the
TABLE I THE MATERIAL AND STRUCTURAL PARAMETERS OF MPP ABSORBERS

\begin{tabular}{|l|c|c|c|c|c|c|}
\hline Material & Specimen & $\mathbf{d}(\boldsymbol{\mu m})$ & $\mathbf{t}(\mathbf{m m})$ & $\mathbf{b}(\mathbf{m m})$ & $\mathbf{D}(\mathbf{m m})$ & $\sigma \%$ \\
\hline PU & 1 & 587 & 2 & 3.14 & 20 & 2.74 \\
\hline PDMS & 2 & 138 & 0.242 & 1.10 & 20 & 1.24 \\
\hline PDMS & 3 & 383 & 0.605 & 6.45 & 20 & 0.28 \\
\hline
\end{tabular}

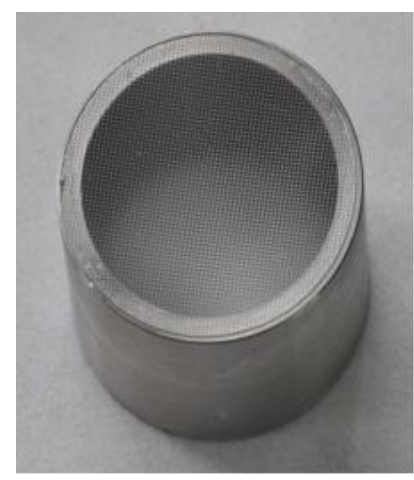

(a)

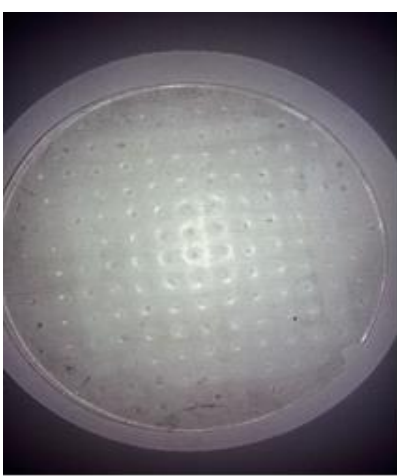

(b)
Figure 3. Photograph of flexible MPPs specimens.

perforation diameter. It can be seen in Table I that the perforation ratio of $1 \#$ specimens is much greater than the flexible MPPs $2 \#$ and 3\#, and the perforation diameter $d$ is much less than $1 \#$ and $2 \#$ specimens' diameters. Comparison experiment of rigid MPPs with or without covering with flexible MPPs or thick membrane will be introduced in next part.

\section{EXPERIMENTAL RESULTS}

The experimental normal absorption coefficients are measured by the impedance tube and standing wave method [11]. We choose 1\# specimens as the base structure, a thick membrane which thickness $t$ is $76 \mu \mathrm{m}$ and two flexible MPPs were respectively placed at the surface of $1 \#$ specimens.

Fig. 4 gives the comparisons of normal absorption coefficients between a rigid MPPs with and without covering with flexible MPPs or thin membrane which is measured at different frequency. Fig. 4(a,b) shows the maximum absorption coefficients of specimen 1\# surfaced with a thin membrane or 2\# flexible MPPs exceeding 0.95 and obtaining wider half-absorption bandwidth in low frequency than 1\# rigid MPPs. This is because of the very high acoustic resistance due to the vibration of plate. However, it can be seen from Fig. 4(c), 1\# rigid MPPs surfaced with $3 \#$ flexible MPPs show higher absorption coefficients exceeding 0.99 in $800 \mathrm{~Hz}$ could be obtained, but with a relatively narrow half-absorption bandwidth. This is due to the fact that lower perforation ratio of $3 \#$ flexible MPPs result in large acoustic mass, which makes the absorption frequency band relatively narrow compared with 1\# rigid MPPs. Based on the Haoyue Ping [12]'s work, the sound absorption peak of flexible MPPs drops from $99.8 \%$ to $94.6 \%$ and shifts to lower frequency from 3 $\mathrm{KHz}$ to $2.5 \mathrm{KHz}$ compared with the same structure rigid MPPs, and the noise absorption bandwidth of flexible MPPs does get widened conspicuously and the sound absorption coefficients at $1.5 \mathrm{KHz}$ and $6 \mathrm{KHz}$ get improved significantly as illustrated in Fig. 4(d). 
Combination a rigid MPPs with a flexible MPPs with high perforation ratio is able to achieve a wider halfabsorption bandwidth in low frequency with the maximum absorption coefficients exceeding 0.95 . In contrast with the rigid MPPs, the absorption performance of traditional MPPs surfaced with flexible MPPs or thin membrane is greatly improved as shown in Fig. 4.

\section{CONCLUSION}

In order to improve the sound absorption capacity of MPPs in low frequency, a rigid MPP absorbers was covered with a membrane or flexible MPPs which was fabricated by MEMS technology or needle-punching and PDMS casting method. Their sound absorption performance experimentally studied in impedance tube in this work. Results shows that combination a rigid MPPs with a flexible MPPs with high perforation ratio is able to achieve a wider half-absorption bandwidth in low frequency with the maximum absorption coefficients exceeding 0.95 , which indicating great potential of combination a rigid MPPs with a flexible MPPs in low frequency noise reduction. Further work is in progress to determine the influence of structure parameters of flexible MPPs on sound absorption performance of the whole structure and even scaling down the perforation diameter of flexible MPPs to micros.

\section{REFERENCES}

[1] Dahyou Maa, "Theory and Design of Microperforated Panel Sound-absorbing Constructions," Scientia Sinica, vol. 18, 1975, pp. $38-50$.

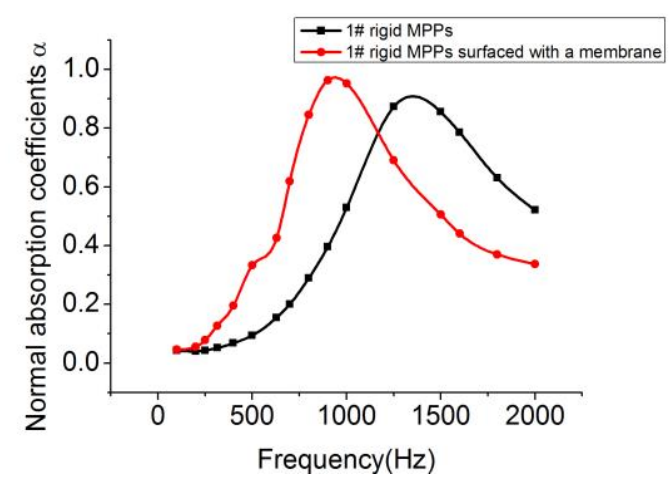

(a)

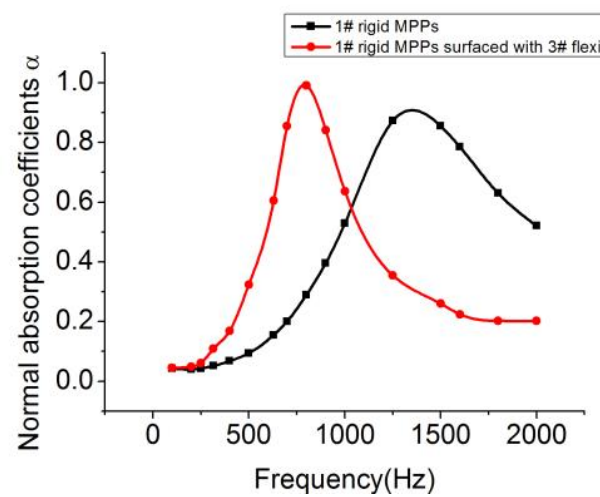

(c)
[2] Y. S. Choy and L. Huang, "Multiple Drumlike Silencer for Low Frequency Duct Noise Reflection," Applied Acoustics, vol. 70, Dec. 2009, pp. 1422-1430.

[3] H. V. Fuchs, U. Ackermann and N. Rambausek, "Non-porous Sound Absorbers for Use in Flue Gas Cleaning Plants," VGB Power Technol, vol. 69, 1989, pp. 965-972.

[4] U. Ackermann and H. V. Fuchs, "Noise Reduction in an Exhaust Stack of a Papermill," Noise Control Engineering Journal, vol. 33, 1989, pp. 57-60.

[5] Shengwo Sheng and Songling Zhao, "Acoustical Characteristics of Perforated Panels with Cover Membranes," Acta Acustica, vol. 19, 1994, pp. 45-52.

[6] J. Kang and H. V. Fuchs, "Predicting the Absorption of Open Weave Textiles and Micro-perforated Membranes Backed by an Air Space," Journal of Sound and Vibration, vol. 220, 1999, pp. 905-920.

[7] Lei Lin, Zuomin Wang and Zaixiu Jiang, "Effect of Soundabsorbing Material on a Microperforated Absorbing Construction," Chinese Journal of Acustics, vol. 30, 2011, pp. 191-202.

[8] Y. J. Qian, D. Y. Kong, Y. Liu, et al, "Improvement of Sound Absorption Characteristics under Low Frequency for Microperforated Panel Absorbers using Super-aligned Carbon Nanotube Arrays," Applied Acoustics, vol. 82, 2014, pp. 23-27.

[9] Jinkyo Lee, George W. Swenson Jr, "Compact Sound Absorbers for Low Frequencies," Noise Control Engineering Journal, vol. 38 , 1992, pp. 109-117.

[10] Y. Y. Lee, E. W. M. Lee and C. F. Ng, "Sound Absorption of a Finite Flexible Micro-perforated Panel Backed by an Air Cavity," Journal of Sound and Vibration, vol. 287, 2005, pp. 227-243.

[11] ISO 10534-1:1996, “Acoustics -- Determination of Sound Absorption Coefficients and Impedance in Ipedance Tubes -- Part 1: Method using Standing Wave Ratio," Geneva, Switzerland, 1996.

[12] Haoyue Ping, Shaohua Wu, Zhan Zhao, et al, "Research about Flexible PDMS Micro-perforated Panel Based on MEMS Silicon Mold," Key Engineering Materials, vol. 609-610, 2014, pp. 701705.

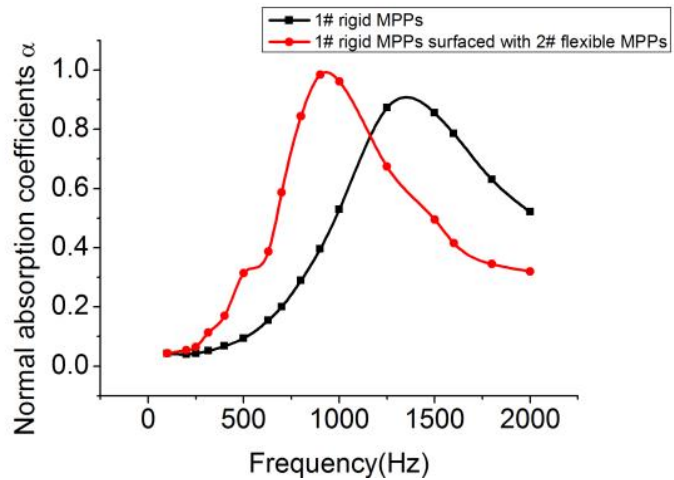

(b)

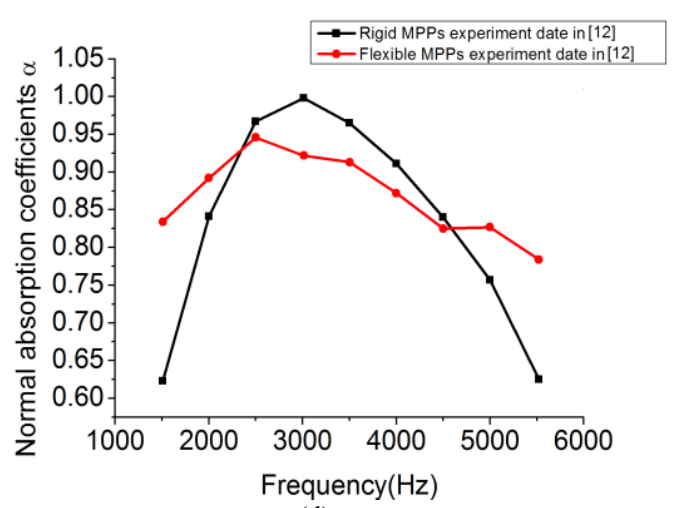

(d)

Figure 4. Normal absorption coefficients of a rigid MPPs with and without covering with flexible MPPs or thin membrane. 